\title{
An examination of semantic radical combinability effects with lateralized cues in Chinese character recognition
}

\author{
Janet Hui-Wen Hsiao \\ University of California, San Diego, La Jolla, California \\ RichaRd SHILlCOCK \\ University of Edinburgh, Edinburgh, Scotland \\ AND \\ Michal LaVidor \\ University of Hull, Hull, England
}

\begin{abstract}
Auclair and Siéroff (2002) examined lateralized cuing effects in the identification of centrally presented letter strings and reported no cuing effects for short word stimuli. They argued for a redistribution of attention over the entire word for short familiar words. We explored cuing effects with Chinese phonetic compounds, which can be considered extreme examples of short words, in a character-level semantic judgment task. When the semantic radical position was placed on the left of the characters, strong radical combinability and semantic transparency effects were observed. There was also a significant interaction between cue position (left vs. right) and radical combinability: A left cue facilitated semantic judgment of characters with small radical combinability more than did a right cue. This behavior reflects the information profile of Chinese phonetic compounds. Semantic radicals with small combinability are more informative than those with large combinability in determining the meaning of the whole character; they therefore benefit more from a left than a right cue. A mechanism redistributing attention over the whole of the character was not in evidence at the level of semantic processing.
\end{abstract}

A fixated four-letter word may typically occupy $1^{\circ}$ of visual angle; selective attention to particular parts of a fixated word is therefore feasible, even for short alphabetic words, making the role of selective attention in reading an important research topic (e.g., Stolz \& McCann, 2000). Auclair and Siéroff (2002) reported an ipsilateral cuing effect on letter identification, with improved processing of the left half of centrally presented strings following a left cue (in comparison with a right cue), and, conversely, improved processing of the right segment following a right cue. Whereas this cuing effect was obtained for pseudowords and nonwords of various lengths, it was only observed for real words of more than nine letters, or for real words of short exposure duration (Auclair \& Siéroff, 2002). Their interpretation was that the weaker cuing effects for words was due to a redistribution of attention over the entire letter string, which benefits familiar words but not nonwords (Besner, Stolz, \& Boutilier, 1997; Brunn \& Farah, 1991).

In the present article, the Posner paradigm (Posner, 1980) was used to explore the role of spatial attention in reading. We present an investigation of lateralized cuing effects for recognition of familiar orthographic strings that extends the
Auclair and Siéroff (2002) paradigm. Instead of letter string identification, the task under examination was a semantic judgment task, which engages deeper cognitive processing levels. The present experiment thus enabled us to examine the extent to which the redistribution of attention over words influenced semantic processing at the lexical level. In contrast to Auclair and Siéroff's materials, the stimuli in the present study were a major type of Chinese character, phonetic compounds. We wished to utilize their distinct square structure and clear separation of different semanticrelated components (or morphemes) within a character.

In Chinese orthography, the dominant structure is the phonetic compound, consisting of a semantic radical and a phonetic radical. The semantic radical usually implies the meaning of the character, whereas the phonetic radical usually bears information about the pronunciation of the character. Radicals usually can also be stand-alone characters. Most phonetic compounds have a left-right structure, with the semantic radical on the left and the phonetic radical on the right ("SP characters"). SP characters comprise about two thirds of Chinese phonetic compounds (Hsiao \& Shillcock, 2006). The distinct square shape of Chinese SP characters can be thought of as similar to two-letter

J.H.Hsiao, jhsiao@cs.ucsd.edu 
words in alphabetic languages, but with a more elaborate alphabet. More specifically, Chinese SP characters consist of semantic-related components, the radicals, that are orthographically more condensed and integral than those of English words. In contrast to English words, the two radicals of a Chinese SP character are separate morphemes and may be separately cued through lateralized cuing manipulation. According to Auclair and Siéroff (2002), lateralized cuing effect was not observed for short English words in an identification task because of a redistribution of attention over the entire letter string. Therefore, Chinese SP characters provide an important test case for examining whether lateralized cues are able to influence semantic processing for short words through selectively cuing different morphemes.

In Chinese phonetic compound recognition, the effect of radical combinability - the number of combinations that a radical enters into to form characters (Feldman \& Siok, 1999) - has long been a controversial issue. Previously, Taft and Zhu (1997) showed that combinability of right radicals influenced character decision time, whereas combinability of left radicals did not. However, Taft and Zhu did not control the function of the radicals - that is, whether the radicals were semantic or phonetic. Feldman and Siok, arguing that this confounded relationship between radical position and function might contaminate the effect of combinability, distinguished between radical position and function by manipulating semantic radical combinability and position. In contrast to Taft and Zhu's findings, they found that high radical combinability of semantic radicals facilitated performance but only when the radicals were on the left, not on the right.

Chen and Weekes (2004) recently presented results of an experiment with 70 participants to examine the effects of character type, radical consistency, and radical combinability in character categorization and character decision tasks. According to their definitions, character type (or semantic transparency) is the extent to which the meaning of a whole character is denoted by the semantic radical; radical consistency is the extent to which a semantic radical consistently represents a specific meaning; radical combinability is the total number of characters that share the same semantic radical. Their results showed that for the character decision task there was no effect of character type, radical consistency, or combinability. However, in contrast to Feldman and Siok's (1999) findings, a significant interaction between radical consistency and combinability emerged for pseudocharacters. On the other hand, in the character categorization task, where participants were asked to judge whether the meaning of the target character belonged to a certain semantic category, Chen and Weekes found significant effects of character type and radical combinability; that is, high semantic radical combinability facilitated semantic category decision, and participants responded to transparent characters faster than they did to opaque characters. Consistency effects were also found to interact with these two variables (Chen \& Weekes, 2004).

The absence of effects in the character decision task is not surprising, considering that the nature of the processing in Chinese character decision tasks is still a con- troversial issue. It is possible that different strategies can be adopted, trading on pronounceability, meaningfulness, and orthographic familiarity. Several priming studies have addressed this issue. According to the direct access hypothesis in Chinese reading research, Chinese characters can be processed for meaning directly from their visual form without activating the phonological system (Shen \& Forster, 1999; Tzeng \& Hung, 1978). Shen and Forster, for example, showed that there was no phonological priming effect in a Chinese character decision task, which can, therefore, be thought of as a semantic processing task. On the other hand, Perfetti and Tan (1998, 1999; Tan \& Perfetti, 1998), the authors of the interactive constituency theory (ICT), argue that the phonological form of a character is unavoidably activated during character identification. In addition, Chen and Weekes (2004) argue that the character decision task shows little or no effect of semantic variables on performance. The variability of the processes involved in the character decision task demonstrates its complexity.

On the other hand, the character categorization task is widely assumed to involve semantic processing of characters, making it an appropriate task to use to examine semantic radical combinability. The results of Chen and Weekes (2004) support this claim; but in their experiment, the position of the semantic radicals in the characters was not completely controlled, and the stimulus materials contained phonetic compounds in several different structures: left-right, top-bottom, and so on. The combinability of each radical was also obtained regardless of position of the radical in a character. In addition, some radicals in Chen and Weekes's study, which were categorized in the large combinability condition, have a combinability value very close to the values in the small combinability condition. ${ }^{1}$

Because of these observations on Chen and Weekes's (2004) materials, we report a more closely controlled examination of semantic radical combinability effects in a Chinese character semantic judgment task. In this experiment, participants were asked to judge whether a given character was semantically transparent or opaque, according to its most frequent meaning. The meaning of a transparent character is directly related to the meaning of its semantic radical. For example, the radical $木$ means tree, wood, or timber; the character 枝, which has 木 as its semantic radical, means branches of a plant, and is, therefore, a transparent character. In contrast, the meaning of an opaque character is not directly related to the meaning of its semantic radical. For example, the character 校, which also has 木 as its semantic radical, means a school, or to proofread, and is, therefore, an opaque character. In the present study, only SP characters were used, with the semantic radical on the left and the phonetic radical on the right. All the characters were left-right structured. Hence, by adopting a lateralized cuing paradigm, we were able to examine how a shift of spatial attention influenced the processing of characters with different semantic radical combinability; potentially, this study could reveal the semantic information profiles of Chinese SP characters through examining their interactions with different-left versus right-lateralized cues. 
In summary, the distinctive structure of Chinese SP characters - a semantic radical on the left and a phonetic radical on the right - is an important test case to examine the influence of spatial attention to short word stimuli at the level of semantic processing, when the word is presented within the foveal area. Because these stimuli are square in shape and might be said to be similar to two-letter words, albeit with an enlarged and more complicated alphabet, the attention redistribution process should therefore be fast, in which case cuing effects are unlikely to be obtained for real characters. Hence, the default prediction, on the basis of Auclair and Siéroff's (2002) data, was that there would be no cuing effects as manifested in the semantic radical combinability effect. If no cuing effects were obtained (as was the case with Auclair and Siéroff's data for short real words), we would have extended the observations of Auclair and Siéroff to a semantic judgment task and provided support for their redistribution theory of attention in lexical processing. Alternatively, if cuing effects were obtained, the data would reveal the extent to which the redistribution of attention influenced Chinese character-level semantic processing and would also potentially reflect the semantic information profiles of Chinese SP characters.

\section{METHOD}

\section{Participants}

The participants were 15 women and 15 men, all right-handed according to the Edinburgh handedness inventory (Oldfield, 1971), all university students in Taiwan (age range, 19-27; mean age, 22 years, 5 months). Participants volunteered, or received a small honorarium for their participation. They were all native Chinese speakers from Taiwan and had similar educational backgrounds. They also had normal or corrected-to-normal vision.

\section{Stimuli}

The material consisted of 192 Chinese phonetic compound characters, with semantic radicals on the left and phonetic radicals on the right. Half of the characters were transparent and the other half were opaque. Within both transparent and opaque groups, half of the characters had a semantic radical with large combinability, and the other half had a semantic radical with small combinability; the characters were divided into four groups, each with 48 characters. All characters were from a medium to high frequency range; according to a frequency count of traditional Chinese character usage reported by Huang (1995), all characters in the materials had a higher frequency than the median frequency, and the average log frequencies of the four groups were all within the 4.4 to 4.9 range. There were no significant frequency differences among the four groups of characters $[F(3,188)=1.34$, n.s. $]$. In order to compare the results of the current study with Chen and Weekes's (2004) study, most characters were selected from Chen and Weekes's materials and converted into traditional forms (since Chen and Weekes used simplified characters); the corresponding transparency was adopted. The additional characters were assessed for transparency by a native speaker of Chinese (J.H.H.) according to a traditional Chinese dictionary (Mandarin Promotion Council, Ministry of Education, R.O.C., 2000). Characters whose transparency was ambiguous, possibly because of more than two high frequency competing meanings, were excluded from the materials.

Combinability of each character was calculated according to a Chinese phonetic compound database, which contains the 2,159 most frequent left-right structured phonetic compounds (Hsiao \& Shillcock, 2006). From this database, we selected 9 semantic radicals from those with largest combinability and 13 semantic radicals from those with smallest combinability. For each semantic radical, the same numbers of transparent and opaque characters that have the given semantic radical were included in the materials; the semantic radicals adopted thus tended to have low consistency, since opaque characters with a highly consistent semantic radical are rare and the same number of transparent and opaque characters are usually difficult to allocate for such semantic radicals.

The semantic radicals with large combinability were $\{$ (人), 口, 小(心), 木, 注(水), 系, 月(肉), 言, and 金. Those with small combinability were 子, 弓, 㣙, 牛, 田, 冰), 米, 耳, 走, 西, 革, 馬, and 黑. According to an analysis of the Chinese character database, all of the semantic radicals in the large combinability group had a combinability larger than 53; in other words, for any given semantic radical in this group, there were more than 53 left-right phonetic compound characters with this same semantic radical. On the other hand, all of the semantic radicals in the small combinability group had a combinability smaller than 19 .

\section{Design}

The experiment included three within-subject variables: character transparency (transparent vs. opaque), semantic radical combinability (large vs. small), and cue position (no cue, left cue, or right cue). The dependent variables were the response times (RTs, in milliseconds), and the accuracy. The design and control of this experiment were conducted with the E-Prime software (Version 1.1; Psychology Software Tools, Pittsburgh, PA). A PST serial response box was used to collect data.

\section{Cuing Paradigm}

The cues were black solid rectangles as high as the characters and a quarter as wide. Before each character presentation, the cue would appear to the left or to the right of the character, or, with an equal probability, it would not appear. In other words, within each of the four character groups, each cue condition was assigned 16 characters. An orthogonal Latin square design was adopted to counterbalance the various sequences in which different cue positions might occur. The participants were divided into three groups receiving different cue and stimulus combinations.

During the experiment, monocular vision was used; that is, each participant looked at the characters with one preferred eye and had the other eye patched to ensure stable fixation. Characters were all presented in a standard calligraphic font and in the same size. The size of the characters was about $1^{\circ}$ of visual angle, and viewing distance was $58 \mathrm{~cm}$. In the cued trials, the cue was presented $1.5^{\circ}$ away from the fixation point (see Figure 1). These presentation locations were applied to ensure that the presented target word was within the foveal area and with the cue, if it appeared, in the parafoveal region.

\section{Procedure}

During the experiment, characters were presented on the computer screen one at a time, in random order. After each presentation, participants were asked to respond to the characters as quickly and accu-

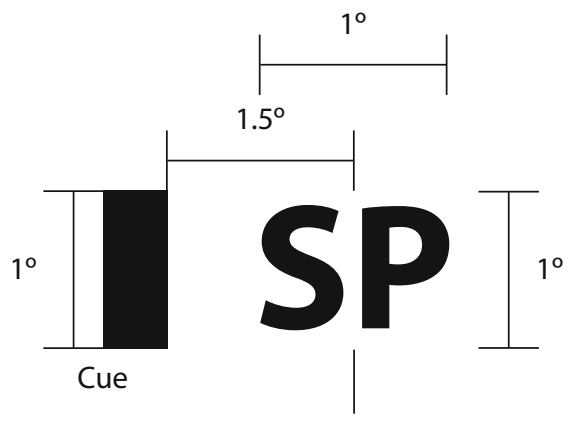

Figure 1. Relative presentation locations of the cue and the character on the screen. 


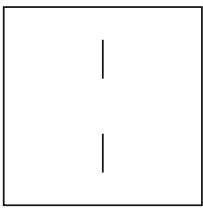

$500 \mathrm{msec}$

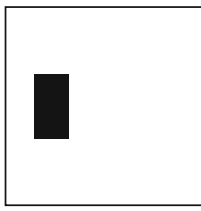

Left Cue/

Right Cue/

No Cue

$80 \mathrm{msec}$

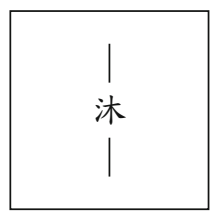

$150 \mathrm{msec}$

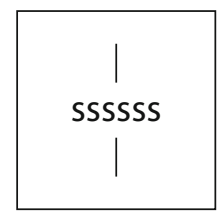

Mask

Figure 2. Timeline of the experiment.

rately as possible by pressing the relevant buttons. If the character was a transparent character according to the most frequent meaning of the character, participants were asked to press, with left and right index finger simultaneously, the inner buttons of a response box with four keys and otherwise to press the outer buttons with left and right middle finger simultaneously. This design was to avoid any hemispheric bias that might be caused by one-hand responses (Mohr, Pulvermüller, $\&$ Zaidel, 1994). RTs were recorded as the time difference between the presentation of the character and the fastest button response. Each cycle of character presentation started with a 500-msec prompt with two short lines above and below the center of the screen, followed by a brief 80-msec presentation of a cue (or a blank screen, if the trial was a no-cue trial) to the left or right of the center of the screen. After that, the target character would appear in the center of the screen for $150 \mathrm{msec}$, followed by a mask that remained on screen until the participant made a response (see Figure 2 for the timeline of the experiment). The next cycle began immediately after the response.

All the materials were evenly divided into four blocks of 48 characters each. Characters from the four different type groups (transparent, large combinability, etc.) were evenly distributed among the four blocks. Each block also had all three cue location conditions evenly distributed. Characters within each block were presented to participants in a random order.

Participants could take a break after each block until they were ready to continue. The importance of maintaining fixation on the central fixation cross was emphasized. Participants were asked to fixate at the middle of the space between the two short lines all the time during the experiment. This position was very close to the middle of the boundary between the phonetic and semantic radicals in each character presentation. Occasionally, a very small one-digit number, or an English letter, was presented between the two prompting short lines, where participants should have fixated. Participants were asked to respond yes to an English letter or no to a number, a procedure intended to help them fixate at the right place (Brysbaert, 1994). Data from any participant who did not respond to the numbers and letters with acceptable accuracy were rejected. In addition, during the experiment, participants used chin rests, which kept them at a distance of $58 \mathrm{~cm}$ from their screens and prevented head movements. (Note that, despite these stringent measures, because of distractions there could still be shifts in fixation toward the location of the lateralized cues; see also Jordan, Patching, \& Milner, 1998.)

Instructions, including a brief review of the meaning of the semantic radicals used in the materials, were given to each participant before the experiment. A practice session was also provided, consisting of characters with semantic radicals that were different from those in the experimental material. Participants had an opportunity to ask any procedural questions regarding the experiment before the test trials began.

\section{RESULTS}

All participants performed satisfactorily on the digit report task; no participant's data were rejected. The mean correct RTs, mean accuracies, and corresponding stan- dard errors as a function of radical combinability, character type, and cue position are summarized in Table 1 .

For accuracies, a repeated measures ANOVA revealed a main effect of semantic radical combinability $[F(1,29)=$ $\left.25.11, M S_{\mathrm{e}}=1.87, p<.001\right]$, with the accuracy of characters with large radical combinability higher than the accuracy of those with small radical combinability; a main effect of character transparency $\left[F(1,29)=30.19, M S_{\mathrm{e}}=\right.$ $10.26, p<.001]$, with the accuracy of transparent characters higher than the accuracy of opaque characters; a significant interaction between combinability and transparency $\left[F(1,29)=30.19, M S_{\mathrm{e}}=2.18, p<.001\right]$, with a significant combinability effect among transparent characters $\left[F(1,29)=78.64, M S_{\mathrm{e}}=1.43, p<.001\right]$ but not among opaque characters $[F(1,29)=0.034$, n.s.]. (See Figure 3.) There was also a marginally significant interaction between cue condition (no cue, left cue, and right cue) and semantic radical combinability $[F(2,58)=2.775$, $\left.M S_{\mathrm{e}}=1.94, p=.07\right]$. In a separate analysis examining the interaction between cue position (left vs. right) and other factors, there was a significant interaction between cue position and semantic radical combinability $[F(1,29)=$ 6.05, $\left.M S_{\mathrm{e}}=1.27, p<.05\right]$. (See Figure 4.) Compared with the results in the right cue condition, the left cue significantly improved participants' accuracy with characters

Table 1

Mean Accuracy and Response Times (RTs, in Milliseconds), With Standard Errors, As a Function of Character Type, Radical Combinability, and Cue Position

\begin{tabular}{|c|c|c|c|c|c|}
\hline \multirow{2}{*}{$\begin{array}{c}\text { Character } \\
\text { Type }\end{array}$} & \multirow[b]{2}{*}{ Combinability } & \multicolumn{2}{|c|}{ Acc. } & \multicolumn{2}{|c|}{ RT } \\
\hline & & $\%$ & $\overline{S E}$ & $M$ & $\overline{S E}$ \\
\hline \multicolumn{6}{|c|}{ No-Cue Condition } \\
\hline \multirow[t]{2}{*}{ Transparent } & Large & 96 & 1 & 854 & 35 \\
\hline & Small & 86 & 2 & 930 & 29 \\
\hline \multirow[t]{2}{*}{ Opaque } & Large & 79 & 3 & 1,126 & 44 \\
\hline & Small & 78 & 3 & 1,161 & 52 \\
\hline \multicolumn{6}{|c|}{ Left-Cue Condition } \\
\hline \multirow[t]{2}{*}{ Transparent } & Large & 95 & 1 & 841 & 28 \\
\hline & Small & 88 & 2 & 928 & 33 \\
\hline \multirow[t]{2}{*}{ Opaque } & Large & 77 & 2 & 1,105 & 51 \\
\hline & Small & 81 & 2 & 1,149 & 58 \\
\hline \multicolumn{6}{|c|}{ Right-Cue Condition } \\
\hline \multirow[t]{2}{*}{ Transparent } & Large & 97 & 1 & 840 & 27 \\
\hline & Small & 86 & 1 & 945 & 37 \\
\hline \multirow[t]{2}{*}{ Opaque } & Large & 77 & 2 & 1,097 & 46 \\
\hline & Small & 77 & 2 & 1,199 & 60 \\
\hline
\end{tabular}




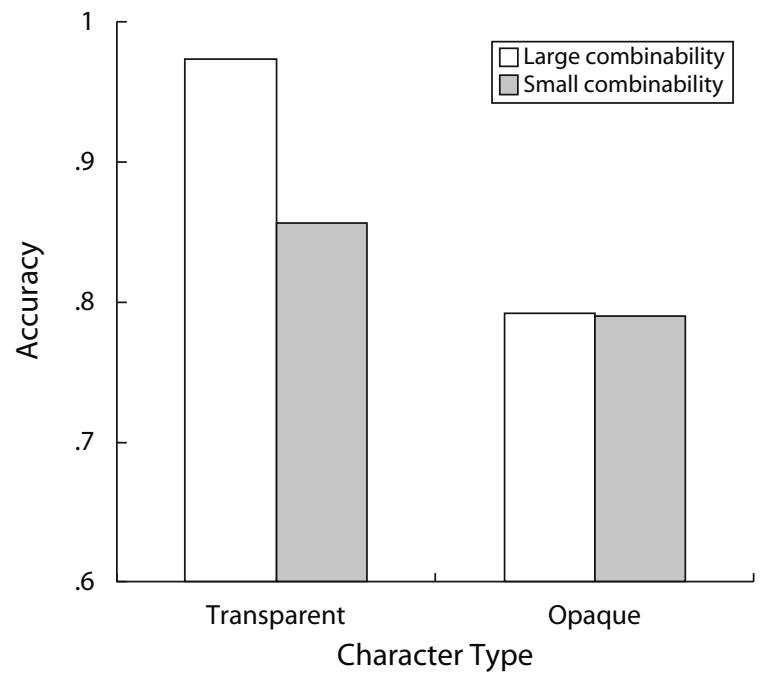

Figure 3. Interaction between character type and semantic radical combinability for accuracy.

with small radical combinability, thereby eliminating the combinability effect [left cue condition, $F(1,29)=1.51$, n.s.; right cue condition, $F(1,29)=25.60, M S_{\mathrm{e}}=1.058$, $p<.001]$.

For RTs, there was a main effect of combinability $\left[F(1,29)=46.40, M S_{\mathrm{e}}=10,864.03, p<.001\right]$, with responses to characters with large radical combinability faster than responses to those with small radical combinability; a main effect of character transparency $[F(1,29)=91.81$, $\left.M S_{\mathrm{e}}=61,274.48, p<.001\right]$, with responses to transparent characters faster than responses to opaque characters. The interaction between combinability and transparency was not significant $[F(1,29)=1.35, p>.05]$. There was a significant interaction between cue condition (no cue, left cue, and right cue) and semantic radical combinability

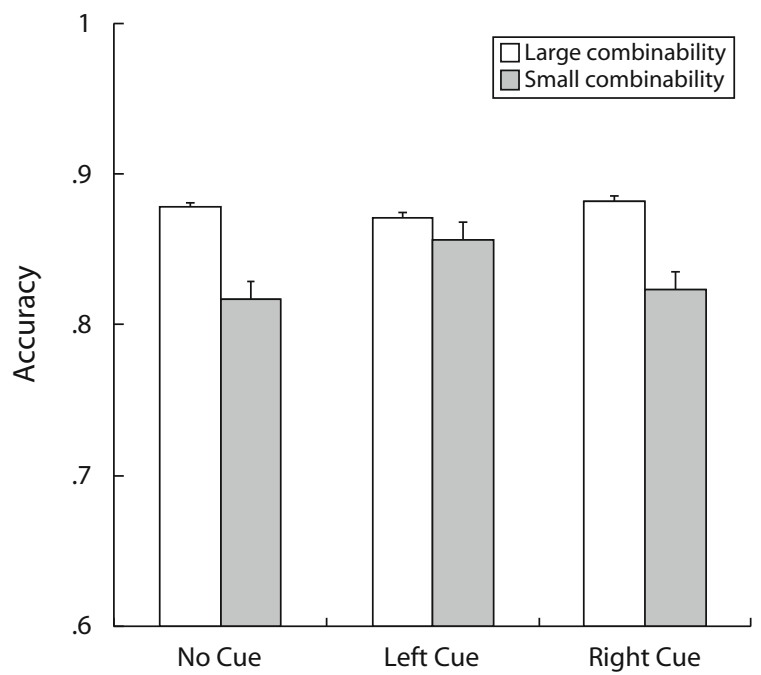

Figure 4. Mean accuracies as a function of semantic radical combinability in different cue conditions. The error bars show standard errors.
$\left[F(2,58)=3.71, M S_{\mathrm{e}}=5,302.33, p<.05\right]$. In a separate analysis examining the interaction between cue position (left vs. right) and other factors, a significant interaction between cue position (left vs. right) and semantic radical combinability was also observed $\left[F(1,29)=5.87, M S_{\mathrm{e}}=\right.$ $3,806.07, p<.05]$. (See Figure 5.) Compared with the right cue condition, characters with small semantic radical combinability were responded to faster in the left cue condition, whereas characters with large semantic radical combinability were responded to slower in the left cue condition.

\section{DISCUSSION}

In a Chinese character semantic judgment task, in which the semantic radical position was controlled to be on the left, a strong combinability effect was observed; large radical combinability facilitated both response speed and accuracy. In addition, the results showed that semantically transparent characters were responded to significantly faster and more accurately than semantically opaque characters. This result may have reflected the difficulty of retrieving the meaning of semantically opaque characters, due to the lack of aid from their semantic radicals or the delay in resolving the conflict between their meaning and the meaning of their semantic radicals. For response accuracy, there was a significant interaction between the semantic radical combinability effect and character semantic transparency; the combinability effect was stronger among semantically transparent characters than among opaque characters. This interaction was not significant in RTs. The combinability effect reported here hence is consistent with Chen and Weekes's (2004) results for their semantic judgment task (i.e., large semantic radical combinability facilitated character categorizations in both RTs and accuracies), but with more control of position of the semantic radicals in the current experiment.

A significant interaction between cue position (left vs. right) and radical combinability was observed in the present experiment: Compared with the right-cue condition, characters with small semantic radical combinability were responded to faster and with higher accuracy in the leftcue condition; in contrast, characters with large semantic radical combinability were responded to slower and with lower accuracy in the left-cue condition (Figures 4 and 5). If the lateralized cues shift attention to the relevant half of the character, and prioritize its processing, such a cue would facilitate the recognition process when it pointed to something informative. According to this view, when the left cue points to a semantic radical with small combinability, it facilitates the semantic judgment, since this semantic radical is informative concerning the meaning of the whole character (given that there are only a small number of characters sharing the same semantic radical). In contrast, the semantic radicals with large combinability are less informative in generating the semantics of the whole character than those with small combinability; since there are a large number of characters sharing the same semantic radical, knowing the semantic radical is not very useful in recognizing the meaning of the whole 


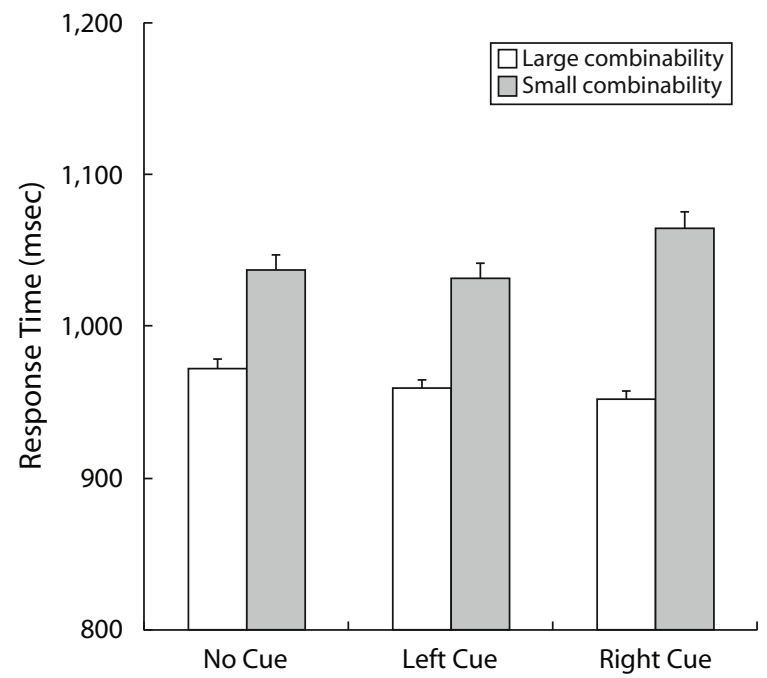

Figure 5. Mean response times as a function of semantic radical combinability in different cue conditions. The error bars show standard errors.

character. In this case, the phonetic radical on the right of a character becomes relatively informative in retrieving the meaning of the whole character. Hence, the right cue somewhat facilitates judgments, because it points to the relatively informative phonetic radical on the right (see Figures 4 and 5).

According to Auclair and Siéroff (2002), because the redistribution process is fast, the redistribution of attention over words makes lateralized cues ineffective with short words. In their experiments, the shortest words used were six letters in length. In the current experiment, we used Chinese SP characters, which may be thought of as being like two-letter words (albeit from a language with a very large alphabet) and served as an extreme case of short words. Hence, cuing effects in character identification were unlikely to be observed, if the processing of Chinese and English were comparable. The cuing effects we obtained in this semantic judgment task may suggest that lateralized cues are more effective at the level of semantic processing than in identification tasks. An alternative explanation is that the complexity of Chinese characters means that the processing of a single phonetic compoundtype character is in fact equivalent to the processing of a long English word, or a shorter English word under degraded conditions, so that cuing may direct attention to different parts of a single character.

In conclusion, this investigation of the semantic combinability effect with lateralized cues has confirmed the facilitatory effects of large semantic radical combinability in a character semantic judgment task, when position of semantic radicals is controlled. Also, it has illuminated the influence of spatial attention on word recognition by showing that cuing effects were still obtained in an extreme case of short words - that is, Chinese characters - in a semantic radical transparency judgment task. The differences from Auclair and Siéroff's (2002) findings are that, whereas the redistribution of attention over the entire stimulus may weaken the effectiveness of lateralized cues for identification of short English words, this redistribution of attention may not influence Chinese character-level semantic processing as much as it would influence English word identification. An alternative explanation is that Chinese characters may be equivalent to long English words (or to degraded shorter English words) in this task. This cuing effect has also reflected the information distribution of Chinese SP characters. For characters with small semantic radical combinability, the semantic radical is informative in retrieving the meaning of the whole character; a left cue will, therefore, direct attention to the semantic radical, and facilitate any associated semantic processing. In contrast, for characters with large semantic radical combinability, the semantic radical is not very useful in determining the meaning of the whole character and the phonetic radical is, therefore, more informative and plays a larger role in identifying the whole character.

In summary, the current experiment has shed further light on the influence of semantic radical combinability on semantic processing of Chinese phonetic compounds, and we have provided a novel information-based analysis of the role of radical combinability.

\section{AUTHOR NOTE}

We thank the BBSRC (U.K.), the European Commission Research Training Network, the Royal Society, the ESRC (U.K.), the Chiang Ching-Kuo Foundation for International Scholarly Exchange (Taiwan), and the NIH (Grant MH57075 to G. W. Cottrell). We also thank two anonymous referees for helpful comments. Correspondence concerning this article should be addressed to J. H. Hsiao, Department of Computer Science \& Engineering, University of California San Diego, 9500 Gilman Drive \#0404, La Jolla, CA 92093-0404 (e-mail: jhsiao@cs.ucsd .edu).

\section{REFERENCES}

Auclair, L., \& SiÉrofF, E. (2002). Attentional cueing effect in the identification of words and pseudowords of different length. Quarterly Journal of Experimental Psychology, 55A, 445-463.

Besner, D., Stolz, J. A., \& Boutilier, C. (1997). The Stroop effect and the myth of automaticity. Psychonomic Bulletin \& Review, 4, 221-225.

BRUNN, J. L., \& FARAH, M. J. (1991). The relation between spatial attention and reading: Evidence from the neglect syndrome. Cognitive Neuropsychology, 8, 59-75.

BRYSBAERT, M. (1994). Interhemispheric transfer and the processing of foveally presented stimuli. Behavioural Brain Research, 64, 151-161.

Chen, M. J., \& Weekes, B. S. (2004). Effects of semantic radicals on Chinese character categorization and character decision. Chinese Journal of Psychology, 46, 181-196.

Feldman, L. B., \& Siok, W. W. T. (1999). Semantic radicals in phonetic compounds: Implications for visual character recognition in Chinese. In J. Wang, A. W. Inhoff, \& H.-C. Chen (Eds.), Reading Chinese script: A cognitive analysis (pp. 19-35). Mahwah, NJ: Erlbaum.

HARBAUGH, R. (1996). Chinese characters and culture. Retrieved Aug. 25, 2004, from www.zhongwen.com.

Hsiao, J. H., \& Shillcock, R. (2006). Analysis of a Chinese phonetic compound database: Implications for orthographic processing. Journal of Psycholinguistic Research, 35, 405-426.

Huang, S. K. (1995). Frequency counts of BIG-5 Chinese characters appeared on Usenet newsgroups during 1993-1994. Retrieved March 16, 2004, from www.geocities.com/hao510/charfreq/.

Jordan, T. R., Patching, G. R., \& Milner, A. D. (1998). Central fixations are inadequately controlled by instructions alone: Implications for studying cerebral asymmetry. Quarterly Journal of Experimental Psychology, 51A, 371-391.

Mandarin Promotion Council, Ministry of Education, R.O.C. 
(2000). Dictionary of Chinese character variants. Retrieved Sept. 25, 2004, from http://140.111.1.40/

Mohr, B., Pulvermüller, F., \& Zaidel, E. (1994). Lexical decision after left, right, and bilateral presentation of function words, content words and non-words: Evidence for interhemispheric interaction. Neuropsychologia, 32, 105-124.

OLDFIELD, R. C. (1971). The assessment and analysis of handedness: The Edinburgh inventory. Neuropsychologia, 9, 97-113.

Perfetti, C. A., \& TAn L. H. (1998). The time course of graphic, phonological, and semantic activation in Chinese character identification. Journal of Experimental Psychology: Learning, Memory, \& Cognition, 24, 101-118.

Perfetti, C. A., \& Tan, L. H. (1999). The constituency model of Chinese word identification. In J. Wang, A. W. Inhoff, \& H.-C. Chen (Eds.), Reading Chinese script: A cognitive analysis (pp. 115-134). Mahwah, NJ: Erlbaum.

Posner, M. I. (1980). Orienting of attention. Quarterly Journal of Experimental Psychology, 32, 3-25.

Shen, D., \& Forster, K. I. (1999). Masked phonological priming in reading Chinese words depends on the task. Language \& Cognitive Processes, 14, 429-459.

Stolz, J. A., \& McCanN, R. S. (2000). Visual word recognition: Reat- tending to the role of spatial attention. Journal of Experimental Psychology: Human Perception \& Performance, 26, 1320-1331.

TAFT, M., \& ZHU, X. (1997). Submorphemic processing in reading Chinese. Journal of Experimental Psychology: Learning, Memory, \& Cognition, 23, 761-775.

Tan, L. H., \& Perfetti, C. A. (1998). Phonological codes as early sources of constraint in Chinese word identification: A review of current discoveries and theoretical accounts. Reading \& Writing, 10, 165-220.

Tzeng, O. J. L., \& Hung, D. L. (1978). Reading the Chinese character: Some basic research. Acta Psychologica Taiwanica, 20, 45-49.

\section{NOTE}

1. For example, the radicals 袨) and 王 (玉) were categorized as a small combinability group, whereas $犭$ (犬) and 禾 were categorized as a large combinability group. However, according to an online Chinese character dictionary (Harbaugh, 1996), the combinability of these radicals is 衣 (49), 玉 (45), 犬 (37), and 禾 (40).

(Manuscript received March 23, 2005; revision accepted for publication August 4, 2006.) 